\title{
Motivos de lesões renais em pacientes que realizam hemodiálise
}

\author{
Reasons for kidney injuries in patients who perform dialysis
}

Causas de lesiones renales en pacientes que realizan diálisis

\begin{abstract}
Beatriz Maria dos Santos Santiago Ribeiro ${ }^{1 *}$, Silvia Rocha de Souza ${ }^{2 *}$, Victor Hideaki Goto Hiraí ${ }^{3}$ Flávio de Paula Neto de Moura ${ }^{4}$
\end{abstract}

\begin{abstract}
RESUMO
Objetivo: Identificar as causas de lesão renal em pacientes que realizam hemodiálise em uma Clínica do Rim no norte do Paraná. Método: Estudo transversal, de caráter descritivo com abordagem qualiquantitativa através de questionário composto por 16 questões à pacientes que realizam hemodiálise na Clínica do Rim no município situado no norte do Paraná. Resultados: Observou-se que $60 \%$ dos pacientes eram do gênero masculino, com idade acima de 51 anos de idade $(66,67 \%)$, média de idade do diagnóstico da doença renal. As causas podemos citar histórico pessoal e familiar de diabetes mellitus com 6,67\%, hipertensão arterial sistêmica com $46,67 \%$. Diabetes e Hipertensão com $26,66 \%$, doença renal com $6,67 \%$, uso de medicação para essas doenças de base por um período superior há 16 anos e após o tratamento hemodialítico com $40 \%$. Ressalta-se a redução de peso após tratamento. Verifica-se que pacientes submetidos ao tratamento inicial de hemodiálise procuram modificar o estilo de vida a fim de melhorar sua saúde. Conclusões: Percebe-se que as causas de lesões renais nesse estudo foram histórico pessoal e familiar de diabetes mellitus, hipertensão arterial sistêmica e, utilização de medicação para essas doenças de base por um período superior à 16 anos. Acredita-se que os resultados obtidos possam contribuir para pesquisas futuras. Desse modo, foi concretizado o objetivo inicial, enfatizando-se identificação das causas de lesão renal.
\end{abstract}

Descritores: Nefrologia, Diálise renal, Enfermagem em nefrologia.

\begin{abstract}
Objective: To identify the causes of renal injury in patients who perform dialysis in a kidney clinic in northern Paraná. Method: Cross-study of descriptive character with Qualiquantitativa approach through a questionnaire consisting of 16 questions to patients who perform dialysis at the kidney clinic in the municipality located in northern Paraná. Results: it was observed that $60 \%$ of patients were male, aged over 51 years of age $(66.67 \%)$, average age of diagnosis of renal disease. The causes we can cite personal and family history of diabetes mellitus with $6.67 \%$, systemic arterial hypertension with $46.67 \%$. Diabetes and hypertension with $26.66 \%$, renal disease with $6.67 \%$, use of medication for these basic diseases for a period of more than 16 years and after the treatment hemodialysis with $40 \%$. Weight reduction after treatment is emphasized. Patients who undergo initial hemodialysis treatment are found to change their lifestyle in order to improve their health. Conclusions: It is understood that the causes of renal lesions in this study were personal and family history of diabetes mellitus, systemic arterial hypertension, and the use of medication for these basic diseases for a period of more than 16 years. It is believed that the results obtained can contribute to future research. Thus, the initial objective was realized, emphasizing the identification of the causes of renal injury.
\end{abstract}

Key words: Nephrology, Renal dialysis, Nursing in nephrology.

\footnotetext{
${ }^{1}$ Mestranda em enfermagem da Universidade Estadual de Londrina (UEL), bolsista Capes, Califórnia-PR.

*E-mail: beatrizsantiago1994@hotmail.com.

${ }^{2}$ Enfermeira especialista em nefrologia, Apucarana-PR.

${ }^{3}$ Especialista em Medicina do Trabalho, Rolândia-PR.

${ }^{4}$ Enfermeiro especialista em saúde coletiva, Apucarana-PR.
} 


\section{RESUMEN}

Objetivo: identificar las causas de la lesión renal en pacientes que realizan diálisis en una clínica renal en el norte de Paraná. Método: estudio cruzado de carácter descriptivo con aproximación Qualiquantitativa a través de un cuestionario consistente en 16 preguntas a pacientes que realizan diálisis en la clínica renal del municipio ubicado en el norte de Paraná. Resultados: se observó que el $60 \%$ de los pacientes eran varones, mayores de 51 años de edad (66,67\%), edad media de diagnóstico de la enfermedad renal. Las causas podemos citar antecedentes personales y familiares de la diabetes mellitus con $6,67 \%$, hipertensión arterial sistémica con $46,67 \%$. Diabetes e hipertensión con $26,66 \%$, enfermedad renal con $6,67 \%$, uso de la medicación para estas enfermedades básicas por un período de más de 16 años y después de la hemodialisis del tratamiento con el $40 \%$. La reducción del peso después del tratamiento se acentúa. Los pacientes que experimentan el tratamiento inicial de la hemodialisis se encuentran para cambiar su estilo de vida para mejorar su salud. Conclusiones: se entiende que las causas de las lesiones renales en este estudio eran antecedentes personales y familiares de la hipertensión arterial mellitus de diabetes, sistémica, y el uso de la medicación para estas enfermedades básicas por un período de más de 16 años. Se cree que los resultados obtenidos pueden contribuir a la investigación futura. Así, se realizó el objetivo inicial, enfatizando la identificación de las causas de la lesión renal.

Palabras clave: Nefrología, Diálisis renal, Enfermería en nefrología.

\section{INTRODUÇÃO}

Os rins desempenham importantes funções no organismo humano, dentre elas destacam-se: filtração sanguínea, produção de substâncias como eritropoietina e renina, excreção de restos metabólicos (ureia, creatinina e ácido úrico) e substâncias estranhas ao corpo como medicamentos, regulação do equilíbrio hidroeletrolítico, ácido básico e osmolalidade (HALL e GUYTON, 2011).

Sabe-se que milhões de pessoas possuem alguma patologia renal com perda de função dos rins devido problemas como cálculos renais, infecção urinária de repetição, glomerulonefrite, alimentação desbalanceada e utilização de alguns medicamentos (SOCIEDADE BRASILEIRA DE NEFROLOGIA, 2013).

Em inquérito realizado no Brasil em 2013 observou-se um aumento de 3\% ao ano de surgimento de problemas renais e, o uso demasiado de medicações em longo prazo contribui para o surgimento dessas doenças e, a falta de fiscalização e acesso sem restrição à compra de alguns medicamentos (antiinflamatórios, analgésicos) colaboram para o crescimento de pessoas com desordens renais. Nesse ano observou um aumento de pacientes, em relação aos anos anteriores com 100.397 de pessoas, sendo que meado dessa população encontravam-se na região Sudeste. e a taxa de prevalência para o tratamento dialítico no mesmo ano foi de 499 pacientes por milhão da população (SESSO, LOPES, THOMÉ et al., 2014).

Os sinais e sintomas comuns que indicam o desenvolvimento de doença renal são: pressão arterial elevada, edema de membros inferiores e ao redor dos olhos, fraqueza constante, náuseas e vômitos frequentes, disúria, poliúria, hematúria, presença de espuma na urina e dor na região lombar (SOCIEDADE BRASILEIRA DE NEFROLOGIA, 2013). Outras condições associadas às doenças renais são: anemia, devido insuficiência de eritropoietina, a qual deve ser reposta subcutaneamente conforme prescrição médica (FERREIRA, 2013); distúrbios ósseos e minerais, como a queda dos níveis de cálcio e vitamina D, os quais devem ser repostos exogenamente e, aumento de fósforo, paratormônio e potássio, podem ser tratados com agentes quelantes (HALL e GUYTON, 2011).

Como forma de tratamento das doenças renais tem-se o controle das doenças de base como diabetes e hipertensão arterial, melhoria do estilo de vida e hábitos alimentares, diálise e terapia medicamentosa (SOCIEDADE BRASILEIRA DE NEFROLOGIA, 2015).

Quando a doença renal progride em estágios avançados apesar do tratamento conservador, o paciente deve ser preparado para o tratamento de diálise ou transplante renal (PERES, PASSARINI, BRANCO et al., 2014). A diálise peritoneal ocorre dentro do corpo do paciente, com auxílio de um filtro natural como substituto da função renal. Uma solução de diálise é infundida e permanece por tempo determinado na cavidade peritoneal (através de cateter implantado no abdômen, próximo ao umbigo), sendo drenada posteriormente; 
essa solução entra em contato com o sangue e remove as substâncias acumuladas como ureia, creatinina e potássio, bem como o excesso de líquido que não está sendo eliminado pelos rins (PEREIRA, RIBEIRO, RUAS et al., 2014).

A hemodiálise é um procedimento através do qual um aparelho recebe o sangue do paciente através de um acesso vascular (cateter ou fístula arteriovenosa) e, depois é impulsionado por uma bomba até o filtro de diálise (dialisador). No dialisador o sangue é exposto à solução de diálise (dialisato), através de uma membrana semipermeável que retira o líquido e as toxinas em excesso do sangue e o devolve limpo para o paciente pelo acesso vascular (FERNANDES. MEDEIROS, MACEDO et al., 2014; SESSO, LOPES, THOMÉ et al., 2014).

O transplante renal, por sua vez, é a última opção de tratamento para pacientes com doença renal crônica avançada, o rim implantado no paciente passa a exercer as funções de filtração e eliminação de líquidos e toxinas, os rins não funcionantes permanecem em seus lugares, a menos que estejam causando infecção ou hipertensão. $O$ transplante renal é considerado a mais completa alternativa de substituição da função renal (CAMPOS, MANTOVANI, NASCIMENTO et al., 2015; KDIGO,2013)

Logo, o profissional de enfermagem é o responsável pelo processo de administração de medicamentos, atividade importante e complexa, onde o enfermeiro presta assistência de qualidade, segurança e eficácia no tratamento a ser realizado, por isso, se faz necessário o conhecimento desse profissional sobre 0 medicamento a ser administrado ao paciente, como reações adversas, mecanismo de ação e interações medicamentosas (CONSELHO FEDERAL DE ENFERMAGEM, 2007).

Sendo assim, o objetivo do presente estudo foi identificar as causas de lesão renal em pacientes que realizam hemodiálise em uma Clínica do Rim no norte do Paraná.

\section{METODOLOGIA}

Estudo transversal, de caráter descritivo com abordagem quali-quantitativa através de questionário composto por 16 questões à pacientes que realizam hemodiálise na Clínica do Rim no município situado no norte do Paraná. Participaram do estudo 15 pacientes. Os critérios de inclusão estabelecidos foram: ser paciente em tratamento hemodialítico, com doenças renais por patologias de base ou por causas desconhecidas, e consentir em participar do estudo. Por sua vez, a ausência do paciente no momento da coleta de dados constituiu critério de exclusão.

Os dados foram coletados, individualmente, no período de dezembro de 2015 a março de 2016, por meio de um formulário sociodemográfico e um questionário composto por 16 questões. Os dados foram submetidos à estatística e organizados em planilhas do programa Excel ${ }^{\circledR}$. Concernente aos aspectos éticos, o projeto de pesquisa foi apreciado e aprovado pelo Comitê de Ética em Pesquisa - CETi da Faculdade de Apucarana FAP, com número do parecer 1.625 .632 de acordo com a Resolução 466 de 12 de dezembro de 2012, do Conselho Nacional de Saúde/Ministério da Saúde, que dispõe sobre pesquisas envolvendo seres humanos.

\section{RESULTADOS E DISCUSSÃO}

De acordo com a análise dos prontuários dos pacientes da Clínica do Rim obtiveram-se os seguintes resultados descritos na primeira tabela referente a dados sociodemográficos e a segunda refere-se aos dados clínicos, como observamos nas tabelas 1 e 2.

Neste estudo a maioria dos pacientes hemodialíticos possui idade superior a 51 anos $(66,67 \%)$, semelhantemente ao observado por Alves (2014); em estudo sobre doença renal policística autossômica dominante em pacientes em hemodiálise no sul do Brasil, em que a idade média dos pacientes foi de 53 anos.

Pôde-se observar que a maioria dos pacientes com problemas renais da Clínica do Rim são homens (60\%), diferente do encontrado por Campos, Mantovani, Nascimento et al., (2015); em avaliação sobre representações sociais sobre o adoecimento de pessoas com doença renal crônica, onde a predominância de pacientes renais era do sexo feminino. 
Tabela 1 - Representação dos dados sociodemográficos

\begin{tabular}{lcc}
\multicolumn{1}{c}{ Variável } & FA & FR \\
\hline Idade & 0 & $0 \%$ \\
Abaixo de 19 anos & 0 & $0 \%$ \\
20 anos aos 30 anos & 2 & $13,33 \%$ \\
31 anos aos 40 anos & 3 & $20,00 \%$ \\
41 anos aos 50 anos & 10 & $66,67 \%$ \\
Acima de 51 anos & & \\
\hline Gênero & 6 & $40,00 \%$ \\
Feminino & 9 & $60,00 \%$ \\
Masculino & $\mathbf{1 5}$ & $\mathbf{1 0 0} \%$ \\
\hline Total
\end{tabular}

Fonte: Próprio autor.

Tabela 2 - Dados clínicos

\begin{tabular}{|c|c|c|}
\hline Variável & FA & FR \\
\hline \multicolumn{3}{|l|}{ Doença } \\
\hline Diabetes & 1 & $6,67 \%$ \\
\hline Hipertensão & 5 & $33,33 \%$ \\
\hline Diabetes e hipertensão & 3 & $20,00 \%$ \\
\hline Outra doença & 6 & $40,00 \%$ \\
\hline \multicolumn{3}{|l|}{ Histórico familiar de doença } \\
\hline Diabetes & 1 & $6,67 \%$ \\
\hline Hipertensão & 7 & $46,67 \%$ \\
\hline Diabetes e Hipertensão & 4 & $26,66 \%$ \\
\hline Doença renal & 1 & $6,67 \%$ \\
\hline Nenhuma & 2 & $13,33 \%$ \\
\hline \multicolumn{3}{|c|}{ Idade de diagnóstico da doença renal } \\
\hline 19 a 29 anos & 3 & $20,00 \%$ \\
\hline 30 a 40 anos & 1 & $6,67 \%$ \\
\hline 41 a 51 anos & 4 & $26,66 \%$ \\
\hline Acima de 51 anos & 7 & $46,67 \%$ \\
\hline \multicolumn{3}{|c|}{ Doença renal apresentada pelo indivíduo } \\
\hline IRC & 11 & $73,33 \%$ \\
\hline Glomerulonefrite & 4 & $26,67 \%$ \\
\hline \multicolumn{3}{|l|}{ Uso de algum tipo de medicação } \\
\hline Sim & 14 & $93,33 \%$ \\
\hline Não & 1 & $6,67 \%$ \\
\hline \multicolumn{3}{|l|}{ Período de utilização de medicação } \\
\hline 1 a 5 anos & 1 & $6,67 \%$ \\
\hline 6 a 10 anos & 4 & $26,66 \%$ \\
\hline 11 a 15 anos & 3 & $20,00 \%$ \\
\hline Acima de 16 anos & 6 & $40,00 \%$ \\
\hline Nenhum & 1 & $6,67 \%$ \\
\hline
\end{tabular}




\section{Continuação da Tabela 2.}

\begin{tabular}{|c|c|c|}
\hline Variável & FA & FR \\
\hline \multicolumn{3}{|c|}{ Tratamento medicamentoso após tratamento hemodiálitico } \\
\hline Sim & 4 & $26,67 \%$ \\
\hline Não & 11 & $73,33 \%$ \\
\hline \multicolumn{3}{|l|}{ Primeiro tratamento da doença renal } \\
\hline Medicamentos e Mudança de hábitos & 5 & $33,33 \%$ \\
\hline Diálise peritoneal & 1 & $6,67 \%$ \\
\hline Hemodiálise & 9 & $60,00 \%$ \\
\hline \multicolumn{3}{|l|}{ Variável (Tratamento atual da doença renal) } \\
\hline Diálise peritoneal & 0 & $0 \%$ \\
\hline Hemodiálise & 13 & $86,67 \%$ \\
\hline Transplante & 2 & $13,33 \%$ \\
\hline Outro & 0 & $0 \%$ \\
\hline \multicolumn{3}{|l|}{ Peso antes do tratamento hemodiálitico } \\
\hline $40-49 \mathrm{Kg}$ & 1 & $6,67 \%$ \\
\hline $50-59 \mathrm{Kg}$ & 2 & $13,34 \%$ \\
\hline $60-69 \mathrm{Kg}$ & 3 & $20,00 \%$ \\
\hline $70-79 \mathrm{Kg}$ & 1 & $6,67 \%$ \\
\hline $80-89 \mathrm{Kg}$ & 4 & $26,66 \%$ \\
\hline Acima de $89 \mathrm{Kg}$ & 4 & $26,66 \%$ \\
\hline \multicolumn{3}{|l|}{ Peso após o tratamento hemodiálitico } \\
\hline $40-49 \mathrm{Kg}$ & 2 & $13,33 \%$ \\
\hline $50-59 \mathrm{Kg}$ & 3 & $20,00 \%$ \\
\hline $60-69 \mathrm{Kg}$ & 4 & $26,67 \%$ \\
\hline $70-79 \mathrm{Kg}$ & 1 & $6,67 \%$ \\
\hline $80-89 \mathrm{Kg}$ & 3 & $20,00 \%$ \\
\hline Acima de $89 \mathrm{Kg}$ & 2 & $13,33 \%$ \\
\hline \multicolumn{3}{|c|}{ Presença de hábito vicioso após o tratamento hemodiálitico } \\
\hline Tabagismo & 4 & $26,67 \%$ \\
\hline Uso de bebida alcoólica & 2 & $13,33 \%$ \\
\hline Nenhum & 9 & $60,00 \%$ \\
\hline \multicolumn{3}{|c|}{ Presença de hábito vicioso após o tratamento hemodiálitico } \\
\hline Tabagismo & 0 & $0,00 \%$ \\
\hline Uso de bebida alcoólica & 1 & $6,67 \%$ \\
\hline Nenhum & 14 & $93,33 \%$ \\
\hline Total & 15 & $100 \%$ \\
\hline
\end{tabular}

Fonte: Próprio autor.

$\mathrm{Na}$ pesquisa realizada observa-se uma porcentagem (40\%) de pacientes hemodialíticos por outras patologias, que não são DM e HAS, como: doença autoimune IGA (imunoglobulina A ou doença de Berger); hipertensão gestacional; infecção de repetição; doença congênita renal; uso de anti-inflamatórios e; causa desconhecida.

Diferente do encontrado pelos autores Sesso, Lopes, Thomé et al., (2014); no Relatório do Censo Brasileiro de Diálise Crônica 2012, onde a causa da doença renal primária, foi em sua maioria, hipertensão arterial (34\%), seguida de diabetes (29\%). As outras doenças, apesar de apresentarem maior percentual também apresentam grande variedade. São 6 doenças diferentes que representam essa porcentagem. Logo, se analisarmos de outra forma o presente estudo também apresenta as maiores causas como HAS, seguida 
de DM. Já Gomes (2011); relata em estudo sobre o impacto da insuficiência renal crônica na eficácia de Stends farmacológicos, que $72,8 \%$ dos pacientes são hipertensos e $31,5 \%$ diabéticos.

As doenças crônicas de base, geralmente herança dos familiares, acabam acarretando outras doenças crônicas, como a insuficiência renal crônica (GOMES, 2011). Dois pacientes relataram não haver histórico familiar das doenças descritas anteriormente (DM, HAS e doença renal) e, desconhecem qualquer outra patologia familiar; já a maioria $(46,67 \%)$, apresenta casos de HAS na família, seguido de DM associada à HAS.

Os autores Sesso, Lopes, Thomé et al., (2014) afirmam, o uso demasiado de medicações em longo prazo contribui para desordens renais Nesta pesquisa, a grande maioria dos pacientes $(93,33 \%)$ utilizam medicamentos como atensina, captopril, enalapril, ácido acetilsalicílico, furosemida, propanolol e losartana , essa divergências de medicamentos se dá pelo uso do tratamento de doenças de base como HAS.

Diferentemente, Marquito, Fernandes, Colugnati et al., (2014); em estudo sobre interações medicamentosas potenciais em pacientes com doença renal crônica, observou que os medicamentos mais utilizados pelos pacientes são fenitoína sódica, fenobarbital, carbamazepina e haloperidol (utilizados para tratamento neurológico como epilepsia).

Percebe-se que a utilização medicamentosa acima de 16 anos é bastante frequente $(40 \%)$; o uso prolongado de medicamentos pode estar relacionado com a progressão do desenvolvimento da doença renal, como mostra Bento, Soares, Molin et al., (2015); ao interligar o uso de varfarina com hemorragia glomerular com obstrução mecânica dos túbulos pelos cilindros eritrocitários e/ou diretamente pela ação nefrotóxica dos produtos da degradação dos glóbulos vermelhos.

Esses pacientes requerem um cuidado sobre as prescrições de medicamentos, devido ao comprometimento da filtração e excreção dos mesmos no organismo (MARQUITO, FERNANDES, COLUGNATI et al., 2014).

A maioria dos pacientes (73,33\%) apresentam insuficiência renal crônica, que pode ter tido sua origem devido à rins policísticos, nefropatia, IGA e uma causa desconhecida que provavelmente deve estar relacionado com o uso de anti-inflamatórios por um período de sete anos consecutivos, segundo relato do acompanhante (RIBEIRO, OLIVEIRA, RIBEIRO et al., 2008).

Já Pereira, Ribeiro, Ruas et al. (2014); em estudo relacionado à análise das principais complicações durante a terapia hemodialítica em pacientes com insuficiência renal crônica, observou que as causas da doença renal são nefroesclerose hipertensiva (31,3\%), glomerulonefrite crônica $(24,5 \%)$ e glomerulares $(15,7 \%)$, resultados diferentes dos obtidos com os pacientes da Clínica do Rim de Arapongas.

Neste estudo, 46,76\% dos pacientes foram diagnosticados com lesão renal após os 51 anos de idade; já no estudo de Filho (2013); sobre a progressão da doença renal crônica: experiência ambulatorial em Santarém - PA, a maioria dos pacientes com doença renal foram diagnosticados após os 60 anos de idade. De qualquer maneira, essa questão se deve ao fato de que a evolução da doença renal está ligada à idade do paciente, onde o metabolismo é mais lento e o organismo não predispõe do mesmo ritmo (ROMÃO, 2004).

Como observou-se apenas $26,67 \%$ continuaram com uso de medicamentos após o tratamento hemodialítico, a pergunta 10 do questionário aplicado, onde os medicamentos que os pacientes utilizam são: complexo B, cloridrato de clonidina e losartana. Para um controle de anemia, hipertensão ou hipotensão e controle da diabetes nos pacientes após a hemodiálise, realiza-se procedimentos de aferição de pressão arterial, glicemia capilar e administra-se por via subcutânea a eritropoietina.

Sabe-se que o peso aumentado dos pacientes renais crônicos pode estar relacionado ao edema provocado pela lesão renal (COITINHO, BENETTI, LIAMARA et al., 2015).

Durante a pesquisa foi observado que alguns pacientes tiveram uma redução de 10 à $20 \mathrm{Kg}$ após 30-90 dias do início do tratamento hemodialítico e sentiram melhorias, como diminuição do cansaço e falta de ar; na clínica é realizado mensalmente a mensuração de medidas e massa magra. 
No estudo de Freire (2013); observou-se uma redução média de 1,2 Kg por paciente ao final de cada sessão hemodialítica, o autor relata que essa diminuição de peso não é expressiva para pacientes que não sejam renais, porém, para pacientes renais essa diferença é muito significativa, uma vez que muitos pacientes relataram que ao retirar esse peso no aparelho de hemodiálise, sentiam-se menos cansados e mais dispostos; sintomas que podem estar relacionados ao acúmulo de líquidos de um dia para o outro ou ingestão aumentada de líquidos.

Houve uma porcentagem significativa de $33,33 \%$ de tratamentos alternativos (utilização de medicamentos e mudança de hábitos de vida) como primeiro tratamento da doença renal; porém, a maioria dos pacientes $(60 \%)$ já iniciaram o tratamento da doença renal com a hemodiálise.

Semelhantemente, Xavier, Santos, Almeida et al., (2014); ao analisar as características individuais e clínicas de clientes com doença renal crônica em terapia renal substitutiva, observou que $66,7 \%$ dos pacientes não tiveram oportunidade de realizar outro tratamento além da hemodiálise. Em ambos os casos, o início direto de tratamento com hemodiálise pode se dar ao fato do curto período de tempo entre a descoberta da lesão renal e os danos provocados ao organismo, sendo necessário uma terapia mais intensa para aliviar os sinais e sintomas provocados pela doença.

Esse fato pode-se dar pela demora de um especialista na rede de Serviço Único de Saúde (SUS), ao próprio usuário do serviço que não se atenta aos sinais e sintomas, e por consequência da própria patologia que é silenciosa.

Durante a pesquisa dois pacientes foram submetidos ao transplante renal, sendo um deles realizado em um período de dois meses após o início do tratamento hemodialítico.

A maior porcentagem de tratamento atual para a doença renal $(86,67 \%)$ é a terapia hemodialítica, cujas sessões variam de 3 a 4 horas, dependendo de cada paciente, podendo iniciar com 4 horas e ir regredindo devido ao seu estado nutricional e/ou emocional. Durante a coleta de dados 2 pacientes evoluíram a óbito, um durante a sessão de hemodiálise e, outro devido ao agravamento de DM.

Notar-se nessa pesquisa que $40 \%$ dos pacientes possuíam algum hábito vicioso, porém, após o diagnóstico de lesão renal, a maioria se absteve do vício, restando apenas um paciente que não conseguiu abandonar o hábito de consumir bebida alcoólica.

O hábito vicioso está associado à progressão e complicação da doença renal existente, além do tabagismo estar associado à progressão da doença renal em pacientes com nefropatia, juntamente com o risco aumentado de doença cardiovascular (BASTOS, 2011).

\section{CONCLUSÃO}

Percebe-se que as causas de lesões renais nesse estudo foram histórico pessoal e familiar de diabetes mellitus, hipertensão arterial sistêmica e, utilização de medicação para essas doenças de base por um período superior há 16 anos. Acredita-se que os resultados obtidos possam contribuir para pesquisas futuras. Desse modo, foi concretizado o objetivo inicial, enfatizando-se identificação das causas de lesão renal.

\section{REFERÊNCIAS}

1. ALVES EF. Autosomal dominant polycystic kidney disease in hemodialysis, patients in southern Brazil. Jornal Brasileiro de Nefrologia, 2014.v. 36, n. 1, p. 18-25.

2. BASTOS MG. Interação medicamentosa na doença renal crônica. Jornal Brasileiro de Nefrologia, 2011, v. 36, n. 1, p. 8-9.

3. BENTO CPQ, SOARES M, MOLIN C et al. Relato de caso: Insuficiência renal crônica estádio $5 \mathrm{D}$ em paciente com doença renal crônica medicado com varfarina. Jornal Brasileiro de Nefrologia, 2015. 37(2), 275-278.

4. CAMPOS CGP, MANTOVANI MDF, NASCIMENTO MEB et al. Social representations of illness among people with chronic kidney disease. Revista Gaúcha de Enfermagem, 2015, v. 36, n. 2, p. 106-112, 2015.

5. COITINHO D, BENETTI ERR, LIAMARA DU et al. Intercorrências em hemodiálise e avaliação da saúde de pacientes renais crônicos. Avances en Enfermería, 2015 33(3), 362. 
6. DE ENFERMAGEM, Conselho Federal. Resolução COFEN 311/2007. Aprova a Reformulação do Código de Ética dos Profissionais de Enfermagem. Rio de Janeiro (Brasil): COFEN, 2007.

7. FERNANDES MCD. MEDEIROS ABA, MACEDO BM et al. Prevalence of nursing diagnosis of fluid volume excess in patients undergoing hemodialysis. Revista da Escola de Enfermagem USP, v. 48, n. 3, p. 446-453, 2014.

8. FERREIRA RCS. Bulário explicativo. 2. ed. São Paulo: Rideel, 2013.

9. HALL JE; GUYTON E. Tratado de fisiologia médica, 2011. 12. ed. Rio de Janeiro: Elsevier.

10. MARQUITO AB, FERNANDES NMS, COLUGNATI FAB et al. Identifying potential drug interactions in chronic kidney disease patients. Jornal Brasileiro de Nefrologia, v. 36, n. 1, p. 26-34, 2014.

11. PEREIRA ER, RIBEIRO IML, RUAS EFG et al. Analysis of major complications during hemodialysis therapy in patients with chronic renal failure. Revista de Enfermagem do Centro Oeste Mineiro, v. 4, n. 2, p. 1123-1134, 2014.

12. PERES LAB, PASSARINI SR, BRANCO MFBT et al. Skin lesions in chronic renal dialysis. Jornal Brasileiro de Nefrologia, v. 36, n. 1, p. 42-47, 2014.

13. ROMÃO JE. Doença renal crônica: definição, epidemiologia e classificação. J Bras Nefrol. 2004;26:1-3

14. RIBEIRO RCHM, OLIVEIRA GASAD, RIBEIRO DF.et al. Characterization and etiology of the chronic renal failure in a countryside nephrology unit of São Paulo State. Acta Paulista de Enfermagem, v. 21, n. especial, p. 207-211, 2008

15. SESSO RC, LOPES AA, THOMÉ FS. et al. Inquérito brasileiro de diálise crônica 2013: análise das tendências entre 2011 e 2013. Jornal Brasileiro de Nefrologia, v. 36, n. 4, p. 476-81, 2014.

16. SOCIEDADE BRASILEIRA DE NEFROLOGIA. Censo de diálise SBN 2013. Acesso em, v. 18, 2013.

17. XAVIER BLS, SANTOS I, ALMEIDA R F et al. Individual and clinical characteristics of clients with chronic kidney disease on renal replacement therapy. Revista de Enfermagem UERJ, v. 22, n. 3, p. 314-320, 2014.

18. KDIGO Clinical practice guideline for the evaluation and management of chronic kidney disease. Kidney Int Suppl 2013;3:1-150. 